\title{
NurOwn, phase 2, randomized, clinical trial in patients with ALS
}

\author{
Safety, clinical, and biomarker results
}

James D. Berry, MD, Merit E. Cudkowicz, MD, Anthony J. Windebank, MD, Nathan P. Staff, MD, Margaret Owegi, MD, Katherine Nicholson, MD, Diane McKenna-Yasek, Yossef S. Levy, PhD, Natalie Abramov, MSc, Haggai Kaspi, PhD, Munish Mehra, PhD, Revital Aricha, PhD, Yael Gothelf, PhD, and Robert H. Brown, DPhil, MD

Neurology ${ }^{\circledR}$ 2019;93:e2294-e2305. doi:10.1212/WNL.0000000000008620

\section{Abstract}

\section{Objective}

To determine the safety and efficacy of mesenchymal stem cell (MSC)-neurotrophic factor (NTF) cells (NurOwn ${ }^{\circledR}$, autologous bone marrow-derived MSCs, induced to secrete NTFs) delivered by combined intrathecal and intramuscular administration to participants with amyotrophic lateral sclerosis (ALS) in a phase 2 randomized controlled trial.

\section{Methods}

The study enrolled 48 participants randomized 3:1 (treatment: placebo). After a 3-month pretransplant period, participants received 1 dose of MSC-NTF cells $(n=36)$ or placebo $(n=$ 12) and were followed for 6 months. CSF was collected before and 2 weeks after transplantation.

\section{Results}

The study met its primary safety endpoint. The rate of disease progression (Revised ALS Functional Rating Scale [ALSFRS-R] slope change) in the overall study population was similar in treated and placebo participants. In a prespecified rapid progressor subgroup $(n=21)$, rate of disease progression was improved at early time points $(p<0.05)$. To address heterogeneity, a responder analysis showed that a higher proportion of treated participants experienced $\geq 1.5$ points/month ALSFRS-R slope improvement compared to placebo at all time points, and was significant in rapid progressors at 4 and 12 weeks ( $p=0.004$ and 0.046 , respectively). CSF neurotrophic factors increased and CSF inflammatory biomarkers decreased in treated participants $(p<0.05)$ post-transplantation. CSF monocyte chemoattractant protein-1 levels correlated with ALSFRS-R slope improvement up to 24 weeks $(p<0.05)$.

\section{Conclusion}

A single-dose transplantation of MSC-NTF cells is safe and demonstrated early promising signs of efficacy. This establishes a clear path forward for a multidose randomized clinical trial of intrathecal autologous MSC-NTF cell transplantation in ALS.

\section{Classification of evidence}

This phase II study provides Class I evidence.

\author{
Correspondence \\ Dr. Brown \\ robert.brown@ \\ umassmed.edu
}

\section{RELATED ARTICLE}

\section{Editorial}

Stem cells in amyotrophic lateral sclerosis: Hype or

hope?

Page 1028

\section{MORE ONLINE}

\section{$\rightarrow$ Class of Evidence}

Criteria for rating

therapeutic and diagnostic studies

NPub.org/coe 


\section{Glossary}

$\mathrm{AE}=$ adverse event; ALS = amyotrophic lateral sclerosis; ALSFRS-R = Revised ALS Functional Rating Scale; CHIT-1 = chitotriosidase-1; CI = confidence interval; DSMB = data and safety monitoring board; FDA = Food and Drug Administration; HGF = hepatocyte growth factor; HHD = hand-held dynamometry; LIF = leukemia inhibitory factor; LS = least squares; MCP1 = monocyte chemoattractant protein-1; MSC = mesenchymal stem cell; NTF = neurotrophic factor; SAE = serious adverse event; SDF-1a = stromal cell-derived factor-1a; SVC = slow vital capacity; TEAE = treatment-emergent adverse event; VC = vital capacity; VEGF = vascular endothelial growth factor.

Amyotrophic lateral sclerosis (ALS) is a fatal neurodegenerative disease characterized by degeneration and death of motor neurons in the brain and spinal cord, and results in progressive muscle weakness and respiratory failure. ${ }^{1}$ The complex pathogenesis of ALS, including the prominent role of neuroinflammation, suggests that emerging ALS treatments will need to address multiple interrelated aspects of the disease.

Bone marrow-derived mesenchymal stem cells (MSCs) are multipotent progenitor cells that have demonstrated high therapeutic potential in neurodegenerative disease. MSCs may enhance neurogenesis, modulate neuroinflammation, and contribute to neuroprotection. ${ }^{2}$ We leveraged the therapeutic potential of neurotrophic factors (NTFs) by inducing bone marrow-derived patient autologous MSCs into MSCNTF cells (NurOwn) secreting high levels of multiple NTFs ${ }^{3}$ and with a unique miRNA signature, ${ }^{4}$ demonstrated to be superior to MSC of origin in several models of neurodegenerative diseases.

In open-label trials, the intrathecal transplantation of NurOwn demonstrated safety and tolerability and showed preliminary evidence of efficacy by slowing the rate of decline on the Revised ALS Functional Rating Scale (ALSFRS-R) after transplantation. ${ }^{5}$

The primary objective of this phase 2 double-blind placebocontrolled multicenter trial was to determine the safety of MSC-NTF cells administration via combined IT and IM injections to participants with ALS. Prespecified analyses investigated the effect of MSC-NTF cells on the slope of the ALSFRS-R in the overall population and in a subgroup of rapid progressors with a pretreatment decline of $\geq 2$ ALSFRS$\mathrm{R}$ points. Analyses of CSF pretransplantation and posttransplantation evaluated the effect of the intervention on relevant MSC and ALS disease biomarkers.

\section{Methods}

\section{Primary research question}

We aimed to determine the safety and efficacy of MSC-NTF cells (NurOwn, autologous bone marrow-derived MSCs, induced to secrete NTFs) delivered by combined IT and IM administration to participants with ALS in a phase 2 randomized controlled trial. This phase II study provides Class I evidence that MSC-NTF cell transplantation in ALS is safe.

\section{Standard protocol approvals, registrations, and patient consents}

This randomized, double-blind, placebo-controlled, phase 2 trial included 3 US participating sites: Massachusetts General Hospital (Boston); Mayo Clinic (Rochester, MN); and the University of Massachusetts (Worcester). The study protocol was cleared by the Food and Drug Administration (FDA) and by the institutional review board of each participating site. The study was conducted in Good Clinical Practice compliance (ClinicalTrials.gov identifier NCT02017912).

\section{Participant selection criteria}

At screening, eligible participants aged $18-75$ years had a diagnosis of possible, probable, laboratory-supported, probable, or definite ALS by El Escorial Criteria, ${ }^{6}$ ALSFRS-R $\geq 30$, vital capacity (VC) $\geq 65 \%$ of the predicted normal value for height, age, and sex, and symptom duration of between 1 and 2 years.

Participants were either not receiving riluzole or were on a stable dose for $\geq 30$ days, had the ability to lie flat for the IT cell transplant procedure, and had at least some limb weakness due to ALS. Potential participants were excluded for the following reasons: use of mechanical ventilation; presence of feeding tube; prior treatment with stem cells; pregnancy; exposure to investigational agents or immunosuppressive therapy within 4 weeks of screening; active autoimmune disease or infection (including hepatitis B, hepatitis C, or HIV), cancer within the previous 5 years; unstable psychiatric or medical condition; or clinically significant abnormal safety laboratory values.

\section{Randomization and blinding}

A permuted block randomization stratified by site with a block size of 4 (ratio of 3:1 of active:placebo) was used for eligible participants to receive MSC-NTF cells or placebo (Dulbecco Modified Eagle Medium), administered on a single occasion by combined IT $\left(125 \times 10^{6}\right.$ MSC-NTF cells in a 5 -mL syringe, using a 20-G spinal needle) administration and 24 IM $\left(48 \times 10^{6}\right.$ MSC-NTF cells) injections in $1-\mathrm{mL}$ syringes to a $1.5-\mathrm{cm}$ depth (ensuring that injection is into the muscle) at 24 separate sites on the participant's right upper arm biceps and triceps muscles, according to a predesigned map. After the IT infusions, the cells were chased with CSF withdrawn from the participant prior to the transplant. Participants were asked to maintain a Trendelenburg position for up to 2 hours after the transplantation. Participants, trial investigators, and sponsor personnel were masked to treatment assignment. 
Treatment allocation was assigned at the cell culture manufacturing facility once the clinical site notified the cell culture facility of subject eligibility. None of the study investigators could have been involved in the treatment assignment in any way. The unblinded clinical teams administering the product (anesthesiologists or other teams) did not have any contact with the participants prior to or after product administration.

\section{Study overview}

After an approximate 3-month run-in period, eligible participants were randomized and their bone marrow was aspirated, followed by MSC-NTF cell transplantation 3 to 4 weeks later. For transplantation, all participants were admitted to the hospital for 48 hours of monitoring. Subsequent outpatient visits occurred 2 weeks after transplantation and then every 4 weeks for 6 months (figure 1A).

\section{Bone marrow aspiration}

All participants, regardless of randomization group, underwent aspiration of 50-70 $\mathrm{mL}$ of bone marrow from the iliac crest by a hematologist per medical center standard procedures. The participants' aspirated bone marrow was immediately transferred to the cGMP cell processing facility in a temperaturecontrolled shipping system per manufacturing collection and shipping procedures.

\section{Cell manufacturing and transplantation}

The cell therapy product was manufactured at 2 central FDAcleared cGMP-compliant manufacturing facilities using standard operating procedures. MSCs were isolated from the bone marrow, expanded, and differentiated to secrete NTFs using a culture-based approach. ${ }^{3,5}$ The MSC-NTF cells production was carried out under full environmental control, in good manufacturing practices-compliant cleanrooms. Fresh autologous MSC-NTF cells were released for transplantation when they fulfilled the cell number, viability, safety (sterility, mycoplasma, and endotoxin), potency (using an ELISA assay for NTF secretion), and identity (CD surface markers) release criteria. Potency was determined based on ELISA assays for NTF secretion. Product was transported from the manufacturing facilities to the clinical sites in a validated shipping system at a controlled temperature of $2^{\circ} \mathrm{C}-8^{\circ} \mathrm{C}$ and administered fresh to the participant. Each shipment's temperature was measured by a data logger.

\section{Outcomes}

The primary objective was to examine the safety of MSCNTF cell transplant in patients with ALS. Safety laboratory studies and adverse events (AEs) were collected at each visit, monitored by the site investigator, medical monitor, and the data and safety monitoring board (DSMB), which was independent from the study and sponsor, and analyzed at a central laboratory.

The second objective was to evaluate the efficacy of MSCNTF cell transplantation in ALS. Therefore, ALSFRS-R and slow VC (SVC) were collected at all pretransplant and posttransplant visits (and at weeks 12 and 20 over the telephone) by trained evaluators who were certified by the Outcomes and Monitoring Center for the Northeast ALS Consortium at Barrow Neurological Institute.

Two exploratory objectives were also examined. The first, hand-held dynamometry (HHD), compared muscle strength in the IM transplanted arm to the noninjected arm. The second exploratory objective examined CSF for changes in NTF, biomarker, and microRNA expression between treated and placebo groups and between pretransplant and posttransplant time points. This was added as a protocol amendment after the commencement of treatment and began with the ninth patient.

\section{CSF analyses}

CSF was collected prior to cell administration and 2 weeks post-transplantation by lumbar puncture, immediately centrifuged at $1,750 \mathrm{~g}$ for 10 minutes, aliquoted, and stored at $-80^{\circ} \mathrm{C}$. Twenty-six sample pairs of treated and 9 sample pairs of placebo participants for whom pretransplantation and posttransplantation samples were available were analyzed using a Multiplex immunoassay (customized Procarta immunoassay, Affymetrix, Santa Clara, CA), according to the manufacturer's instructions. Mean fluorescence intensities of analyte-specific immunoassay bead sets were detected by a flow-based Luminex (Austin, TX) 3D suspension array system. Chitotriosidase-1 (CHIT-1) was analyzed by ELISA.

\section{Statistical analysis}

The primary trial outcome was safety, assessed with respect to the incidence of treatment-emergent AEs (TEAEs) and serious AEs (SAEs), laboratory abnormalities, vital signs, ECGs, and physical examinations. AEs were coded using MedDRA.

Secondary endpoints were comparisons of (1) the change in slopes (post-treatment slope through 24 weeks as compared to the pretreatment slope) in ALSFRS-R and SVC between the treatment and placebo groups and (2) the post-treatment slope of decline in the ALSFRS-R and SVC at 24 weeks following transplantation relative to the 12 - to 16 -week baseline period before transplantation (pretreatment slope) in all patients (within each treatment group).

Prespecified efficacy analyses in the statistical analysis plan included comparison of ALSFRS-R slopes post-treatment vs pretreatment between treatment groups using model fit least squares (LS) means as well as responder analyses comparing the MSC-NTF and placebo groups. Responder analyses were prespecified and defined as a $20 \%-30 \%$ improvement in posttreatment slope compared to pretreatment.

This was a phase 2 study for which no formal sample size calculation was performed and the sample size was not based on statistical considerations. The total of 36 treatment and 12 placebo participants are expected to be a sufficient number to 


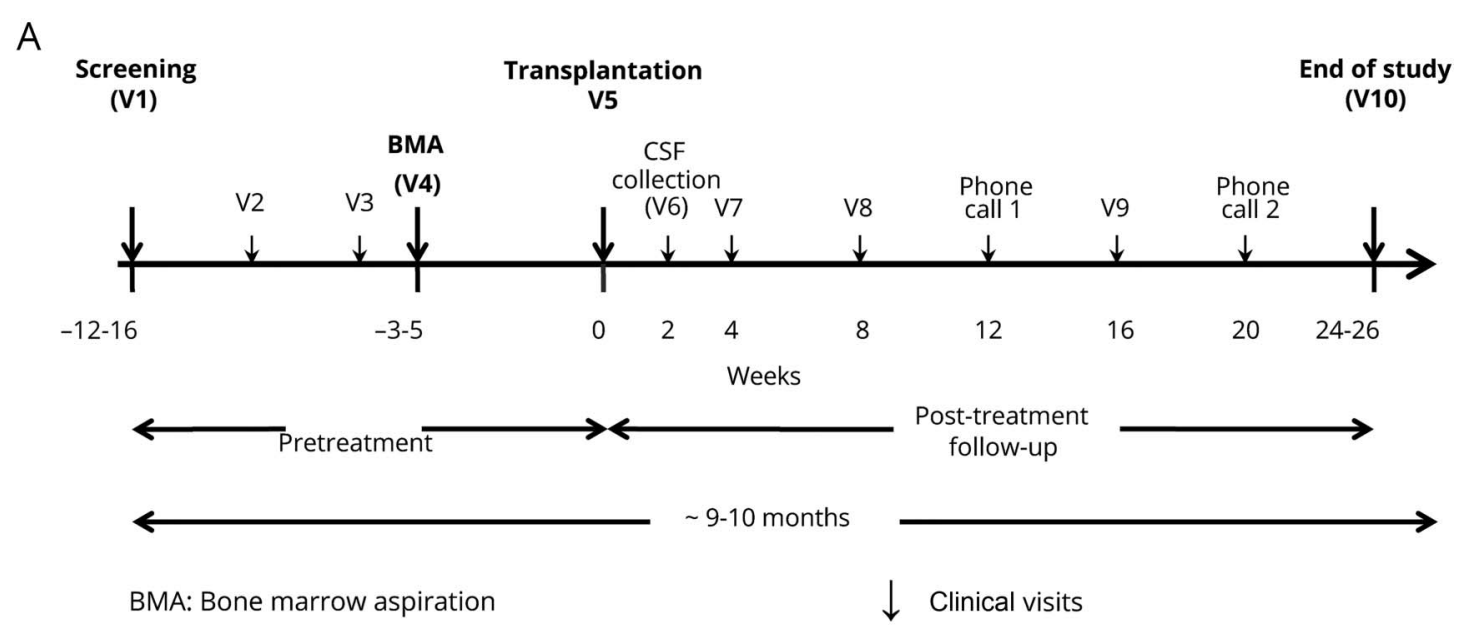

B

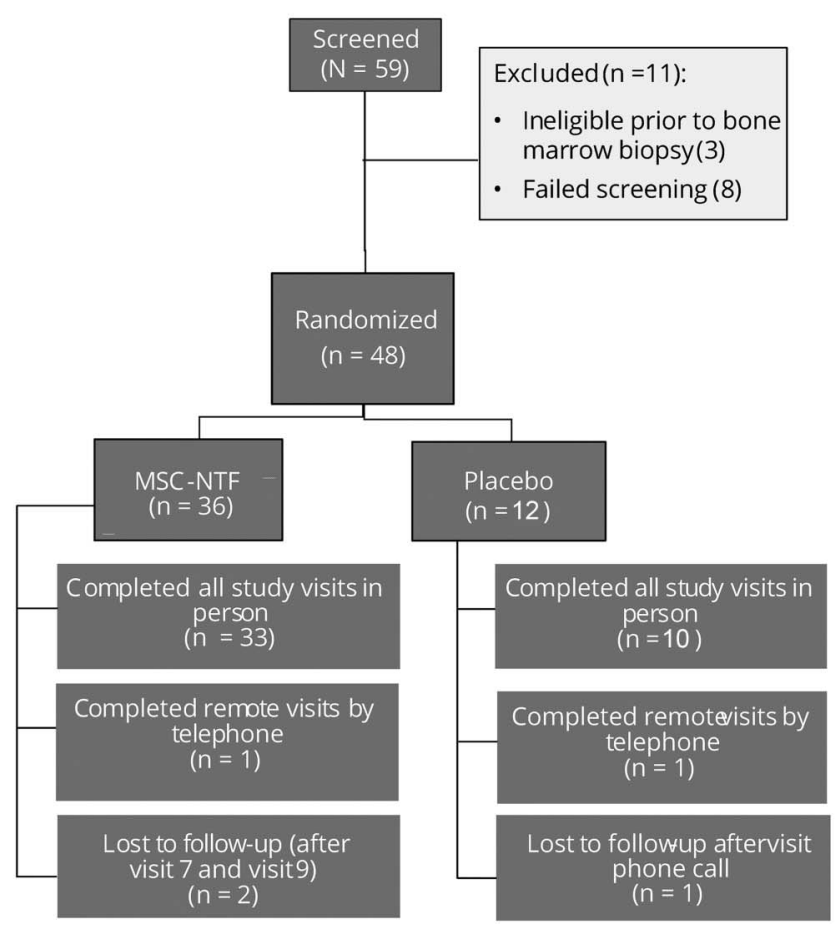

(A) Trial design. (B) CONSORT diagram: participant enrollment, intervention allocation, and follow-up. MSC = mesenchymal stem cell; NTF = neurotrophic factor.

obtain adequate characterization of common TEAEs and to observe trends for treatment effects on the efficacy measures chosen for this study. In addition, with 36 participants in the MSC-NTF treatment group, the study has $95 \%$ probability of seeing AEs that occur at a true rate of $8 \%$ or greater. Therefore, if an AE of a specific type does not occur, there is $95 \%$ probability that the true rate of the AE is less than $8 \%$. Efficacy analyses were not adjusted for multiplicity.

We examined 2 methods for defining cutoffs: (1) defining an exact change in ALSFRS-R slope in points per month and (2) using a percent change in ALSFRS-R post-treatment slope compared to pretreatment slope. For each method, at each follow-up visit we compared the post-transplantation slope with the pretransplantation slope and defined a range of thresholds for responders ( $\geq 0.5$ to $\geq 2.5$ points/month; and $\geq 25 \%$ to $\geq 100 \%$ change); however, analysis of points/month improvement in the ALSFRS-R slope was found to be more accurate, particularly in the case of a zero pretreatment slope, in which case a percent improvement analysis was not feasible. Available data were used to compute slopes, which were assumed at later time points for missing data.

Three prespecified subgroup analyses included ALSFRS-R overall pretreatment score change $(<-2$ points, rapid progressors; or $\geq-2$ points, slow progressors); ALSFRS-R motor 
pretreatment score change ( $<-1$ points vs $\geq-1$ points); and baseline SVC ( $\geq 70 \%)$. Secondary and exploratory objectives included analyses of SVC, HHD, and CSF biomarkers. Statistical significance for the LS means was determined if the $p$ value was $\leq 0.05$ (2-sided) and for responder analyses if the $p$ value was $<0.05$ (2-sided Fisher exact test).

The CSF data were statistically assessed for significance by either Student $t$ test or analysis of variance analysis as applicable. A $p$ value of less than 0.05 was considered statistically significant.

\section{Data availability}

Appropriate anonymized data can be made available to qualified investigators upon submission of an acceptable analysis plan. Proposals should be directed to rkern@brainstorm-cell.com. To gain access, data requestors will need to sign a data access agreement.

\section{Results}

Fifty-nine participants were screened in this trial. There were 8 screen failures, and during the 3 -month run-in period, 3 participants discontinued the study prior to randomization. Forty-eight participants were randomized, of whom 43 completed follow-up (figure 1B).

Treatment arms showed similar baseline characteristics. The majority of the treated participants were male (35 participants, $72.9 \%$ ) and all were white (48 participants, 100\%). The mean age of the participants was 51.1 years (range 26-71; table 1).

\section{Safety}

The trial met its primary safety endpoint; thus the treatment appears to be safe. Forty-three participants (90\%) completed the follow-up period (33 MSC-NTF, 10 placebo). Five participants discontinued in-person follow-up; of these, 2 completed the trial by remote telephone follow-up and were therefore included in all safety and ALSFRS-R analyses (1 MSC-NTF, 1 placebo; figure $1 \mathrm{~B})$. There were no deaths during the study, no treatment-related SAEs, and no AEs that led to dropout. Two participants in the MSC-NTF treatment arm underwent tracheostomy placement for respiratory failure following placement of a feeding tube, approximately 4 months post-treatment.

Over the course of the study, 11 participants-9/36 in the MSC-NTF cells group (25\%) and 2/12 in the placebo group (17\%)-developed 16 SAEs (table 2). Two SAEs occurred after trial entry but prior to treatment. All treatment-emergent SAEs (AEs that occurred after initiation of study treatment) were deemed to be related to ALS disease progression, and none was considered possibly, probably, or definitely related to study treatment. The DSMB did not identify any AEs, laboratory abnormalities (blood hematology, chemistries, urinalysis), or substantial protocol deviations that would be a cause for concern.

Table 3 summarizes the number of treatment-emergent and treatment-related AEs or SAEs that occurred during the study and lists all AEs that occurred in $>15 \%$ of patients from either group. AEs related to the delivery of MSC-NTFs or placebo were transient and included headache (75\% MSC-NTFs; 50\% placebo), fever (33\% MSC-NTFs; 0\% placebo), back pain

Table 1 Demographics and baseline characteristics by treatment group

\begin{tabular}{llll}
\hline \multicolumn{1}{l}{ Sex } & MSC-NTF $(\mathbf{n}=\mathbf{3 6})$ & Placebo $(\mathbf{n}=\mathbf{1 2})$ & All participants $(\mathbf{n}=\mathbf{4 8})$ \\
\hline Male & $25(69.4)$ & $10(83.3)$ & $35(72.9)$ \\
\hline Female & $11(30.6)$ & $2(16.7)$ & $13(27.1)$ \\
\hline Age, $\mathbf{y}$ & $50.3(11.90)$ & $53.5(9.11)$ & $51.1(11.27)$ \\
\hline El Escorial criteria & & & $1(8.3)$ \\
\hline Possible & $3(8.3)$ & $1(8.3)$ & $4(8.3)$ \\
\hline Laboratory-supported probable & $5(13.9)$ & $7(58.3)$ & $6(12.5)$ \\
\hline Probable & $16(44.4)$ & $3(25.0)$ & $23(47.9)$ \\
\hline Definite & $12(33.3)$ & $9.01(4.63)$ & $15(31.3)$ \\
\hline ALS medical history, months since diagnosis & $9.00(5.57)$ & $16.75(3.10)$ & $9.00(5.31)$ \\
\hline ALS medical history, months since first symptoma & $17.62(3.79)$ & $17.40(3.62)$ \\
\hline
\end{tabular}

Abbreviations: ALS = amyotrophic lateral sclerosis; ALSFRS-R = Revised ALS Functional Rating Scale; MSC = mesenchymal stem cell; NTF = neurotrophic factor. Data are presented as $\mathrm{n}(\%)$ or mean $( \pm S D)$. Percentages are based on the number of participants in a given treatment group for the population being analyzed.

a The site of ALS onset was not tracked in the course of the study. 
Table 2 Overall summary of adverse events (AE) and serious AEs (SAEs) by treatment group: safety population

\begin{tabular}{|c|c|c|}
\hline TEAEs & MSC-NTF cells, $n=36, n(\%)$ & Placebo, $n=12, n(\%)$ \\
\hline TEAEs & 585 & 109 \\
\hline Participants with at least 1 TEAE & $36(100)$ & $12(100)$ \\
\hline Treatment-related TEAEs $^{a}$ & 197 & 32 \\
\hline Participants with at least 1 treatment-related TEAE & $35(97.2)$ & $9(75.0)$ \\
\hline Treatment-related serious TEAEs & 0 & 0 \\
\hline Participants with at least 1 treatment-related serious TEAE & 0 & 0 \\
\hline \multicolumn{3}{|l|}{ Participants with TEAEs by maximum severity } \\
\hline Mild & $36(100)$ & $12(100)$ \\
\hline Moderate & $34(94.4)$ & $10(83.3)$ \\
\hline Severe & $3(8.3)$ & $1(8.3)$ \\
\hline Potentially life-threatening & $1(2.8)$ & 0 \\
\hline SAES & 14 & 2 \\
\hline Participants with at least 1 SAE & $9(25)$ & $2(16.6)$ \\
\hline Participants with at least 1 treatment-emergent SAE & $8(22.2)$ & $1(8.3)$ \\
\hline Participants with treatment-related SAE & 0 & 0 \\
\hline Participants with TEAEs resulting in treatment withdrawal & 0 & 0 \\
\hline Participants with TEAEs resulting in withdrawal from study & 0 & 0 \\
\hline Participants with TEAEs resulting in death & 0 & 0 \\
\hline
\end{tabular}

Abbreviations: MSC = mesenchymal stem cell; NTF = neurotrophic factor; TEAE = treatment-emergent adverse event.

Percentages are based on the number of participants in a given treatment group for the population being analyzed. An AE is considered a TEAE if the start date/time of the $\mathrm{AE}$ is on or after the date/time of initiation of cell transplantation or if the severity worsens after the initiation of cell transplantation.

a Treatment-related TEAEs are TEAEs that are considered to have probable, possible, or definite relationship to the study drug.

(72\% MSC-NTFs; 8\% placebo), and injection site bruising (30.6\% MSC-NTF; 25\% placebo).

\section{Efficacy analyses}

\section{ALSFRS-R LS mean slope change}

At screening, ALSFRS-R scores were similar in the MSC-NTF and placebo groups $(38.1 \pm 3.6$ and $38.6 \pm 3.85$, respectively). Pretreatment ALSFRS-R slopes were similar in the 2 groups: -0.7 points/month (MSC-NTF) and -0.6 points/month (placebo). The change in the ALSFRS-R slope posttransplant was +1.7 points/month (MSC-NTF) and -0.4 points/month (placebo) at 2 weeks $(p=0.110)$, and +0.6 points/month (MSC-NTF) and -0.03 points/month (placebo) at 4 weeks $(p=0.368)$. After 8 weeks, the change in slope was similar in both treatment arms (figure $2 \mathrm{~A}$ ).

In the prespecified rapid progressors subgroup (i.e., those with a decline of $>2$ points in ALSFRS-R scores during the 3 -month pretreatment period), there were 15 participants $(42 \%)$ in the MSC-NTF arm and $6(50 \%)$ in the placebo arm. Pretreatment ALSFRS-R slopes were comparable: -1.5
(MSC-NTF) and -1.2 (placebo) $(p=0.232)$. Comparison of LS means of the post-transplant ALSFRS-R slope minus the pretransplantation slope between the MSC-NTF and placebo groups demonstrated a significant improvement in the MSCNTF group at 2 and 4 weeks $(+3.3$ vs $-1.3 ; p=0.021$, and +2.0 vs $-0.1 ; p=0.033$, respectively) and a continued trend for improvement in the MSC-NTF group at all remaining time points (figure $2 \mathrm{~B}$ ).

\section{ALSFRS-R responder analyses}

Using the $\geq 1.5$ point/month improvement criterion (ALSFRS-R slope post-treatment compared to pretreatment slope), a higher proportion of responders were observed in the MSC-NTF cells group compared to placebo at all time points. The difference was significant at week 4 (MSC-NTF $47 \%$, placebo $9 \%, p=0.033$, figure $2 \mathrm{C}$ ).

In the rapid progressors subgroup, a higher proportion of responders ( $\geq 1.5$ points/month ALSFRS-R slope improvement) were observed in the MSC-NTF group compared to the placebo group at all time points; the difference was significant at week 4 ( $80 \%$ vs $0 \%$; $95 \%$ confidence interval [CI] of difference of proportions $59.8 \%-100 \%, p=0.004)$ and 
Table 3 Percentage of participants with treatmentemergent adverse events by trial arm and type $(>15 \%$ in either group)

\begin{tabular}{lll}
\hline Adverse event & MSC-NTF cells & Placebo \\
\hline Headache and procedural headache & 80.6 & 66.7 \\
\hline Back pain & 72.2 & 8.3 \\
\hline Pyrexia & 33.3 & 0 \\
\hline Arthralgia & 33.3 & 0 \\
\hline Injection site pain & 27.8 & 8.3 \\
\hline Constipation & 25 & 8.3 \\
\hline Pain in extremity & 22.2 & 0 \\
\hline Neck pain & 19.4 & 0 \\
\hline Myalgia & 16.7 & 0 \\
\hline Cough & 16.7 & 0 \\
\hline Nausea & 16.7 & 0 \\
\hline
\end{tabular}

Abbreviations: $\mathrm{MSC}=$ mesenchymal stem cell; NTF = neurotrophic factor .

week 12 (53\% vs $0 \%$; $95 \%$ CI of difference of proportions $28.1 \%-78.6 \%, p=0.046$, figure $2 \mathrm{D})$.

\section{Slow vital capacity}

Screening SVC was comparable in the MSC-NTF cells and placebo groups (90.6 \pm 18.1 and $88.7 \pm 8.91$, respectively). Analysis of the changes in post-treatment compared to pretreatment slope and responder analyses with various thresholds showed no significant differences between the 2 treatment groups. No significant treatment effects were noted in the rapid progressors group.

\section{Hand-held dynamometry}

Comparison of muscle strength in the IM transplanted arm to the noninjected arm, as measured by HHD, did not demonstrate significant side-to-side difference in HHD muscle strength score slopes over 24 weeks.

\section{CSF analyses}

CSF was collected pretransplant and 2 weeks post-transplant. Only pretransplant and post-transplant paired samples were analyzed. In total, 26 treated and 9 placebo CSF sample pairs were available for analysis.

\section{Neurotrophic factors}

Levels of NTFs secreted by the MSC-NTF cells in vitro were evaluated in the CSF. Average basal levels of vascular endothelial growth factor (VEGF) were $37.1 \pm 15.8 \mathrm{pg} / \mathrm{mL}$ and 30.6 $\pm 14.4 \mathrm{pg} / \mathrm{mL}$ (mean \pm SEM) in treated and placebo participants, respectively. Post-transplantation, the mean VEGF increase in the treated participants was $629.8 \pm 243.3(p=0.016)$ and $-0.78 \pm 0.9(p=0.45)$ in placebo participants. Basal hepatocyte growth factor (HGF) levels were high (treated participants $391.1 \pm 115.9 \mathrm{pg} / \mathrm{mL}$; placebo participants $448.4 \pm$ $146.3 \mathrm{pg} / \mathrm{mL}$ ). Post-transplantation, HGF levels significantly increased in the MSC-NTF-treated participants (mean change $107.18 \pm 34.4 ; p=0.004)$ while there was no change in those receiving placebo $(18.8 \pm 22.2 ; p=0.42)$. Leukemia inhibitory factor (LIF) was undetectable in the CSF prior to transplantation and significantly increased (12.33 \pm 3.23 ; $p=$ 0.0008 ) post-transplantation in treated participants, while there was no detectable LIF in the placebo group either pretransplantation or post-transplantation (figure $3 \mathrm{~A}$ ).

\section{Inflammatory biomarkers}

Inflammatory biomarkers known to be present in the CSF of patients with ALS, as well as cytokines, chemokines, and cellular factors that reflect key processes relating to immune pathways, CNS inflammation, and cell death, were selected for analysis. Not all biomarkers analyzed were detectable in the CSF.

Basal levels of monocyte chemoattractant protein-1 (MCP-1) were $42.2 \pm 9.4 \mathrm{pg} / \mathrm{mL}$ in treated participants and $40.5 \pm 9.5$ $\mathrm{pg} / \mathrm{mL}$ in the placebo group. Post-transplantation, mean MCP1 levels decreased significantly in the MSC-NTF-treated participants $(-17.3 \pm 1.8 \mathrm{pg} / \mathrm{mL} ; p<0.0001)$, while no significant change was observed in the placebo group $(-0.89 \pm 2.3 \mathrm{pg} / \mathrm{mL}$; $p=0.7)$. The post-transplantation difference between MSCNTF-treated participants and the placebo group was statistically significant $(p<0.0001)$. Average basal levels of stromal cell-derived factor-1a (SDF-1a) were $195.6 \pm 31.1 \mathrm{pg} / \mathrm{mL}$ in MSC-NTF-treated participants and $200.8 \pm 14.9 \mathrm{pg} / \mathrm{mL}$ in the placebo group. Post-transplantation, SDF-1a significantly decreased in the MSC-NTF-treated participants $(-46.6 \pm 12.9$ $\mathrm{pg} / \mathrm{mL} ; p=0.0014)$, while it remained unchanged in the placebo group $(0.27 \pm 5.05 \mathrm{pg} / \mathrm{mL} ; p=0.96)$. The posttransplantation difference between the 2 groups was statistically significant $(p=0.04)$. CHIT-1 demonstrated a small and significant decrease in treated patients $(-1,413 \pm 601 ; p=0.027)$ and a small nonsignificant increase in placebo patients $(6,411 \pm$ 2,137 ) post-transplantation (figure 3B). There was no significant post-transplantation change in the levels of macrophage inflammatory protein- $1 \beta$ or C-reactive protein in either group (data not shown). A trend towards a greater reduction of CSF inflammatory factors was observed in responder patients at some timepoints, which did not reach significance, possibly due to the small sample size.

We also examined the levels of caspase-3, one of the key mediators of apoptosis. Interestingly, caspase- 3 was significantly reduced post-transplant in MSC-NTF-treated participants $(-2.09 \pm 0.4 ; p<0.0001)$ but not in the placebo group $(-0.91 \pm 0.9 ; p=0.35)$. A significant reduction $(p=$ $0.02)$ in responders $(\geq 100 \%$ improvement in ALSFRS-R slope 12 weeks post-transplantation) was observed compared to nonresponders.

Two weeks post-transplant, a statistically significant inverse correlation was observed between VEGF and MCP-1 (correlation $-0.56, p=0.003$, figure $4 \mathrm{~A})$, VEGF and SDF-1a 
Figure 2 Mean change in Revised ALS Functional Rating Scale (ALSFRS-R) slope over time (top) and responder analyses: $\geq 1.5$-point ALSFRS-R slope improvement over the post-treatment follow-up period (bottom)

A. Change from post-treatment to pretreatment slope

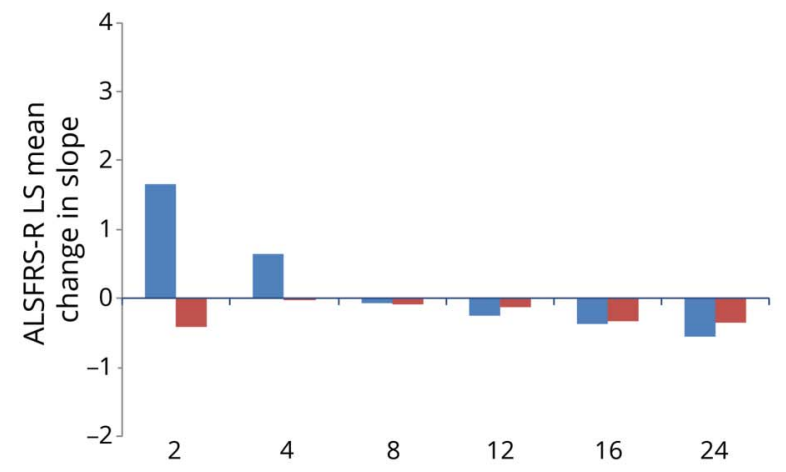

C

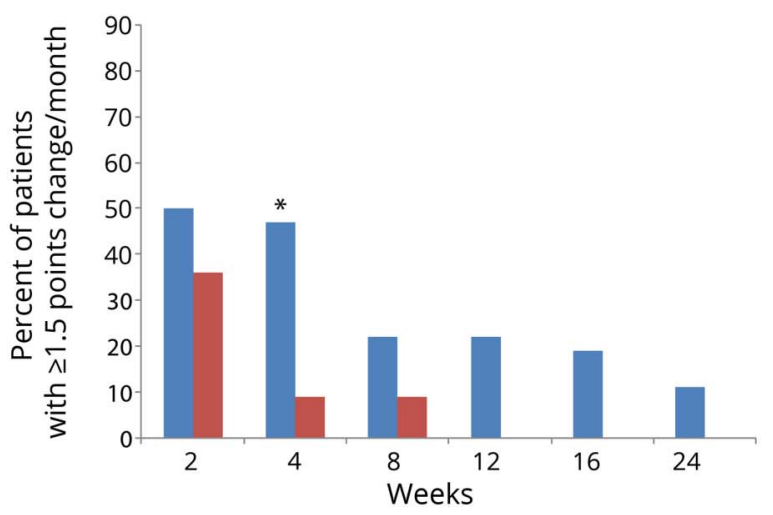

B. Change from post treatment to pretreatment slope

MCS-NTF

- Placebo

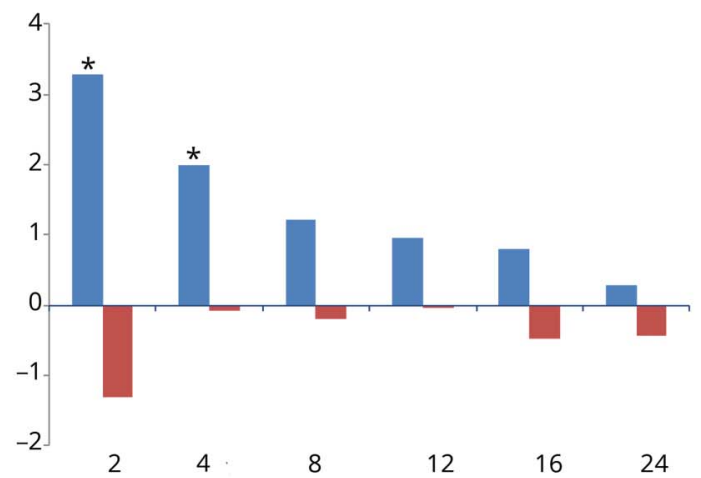

D

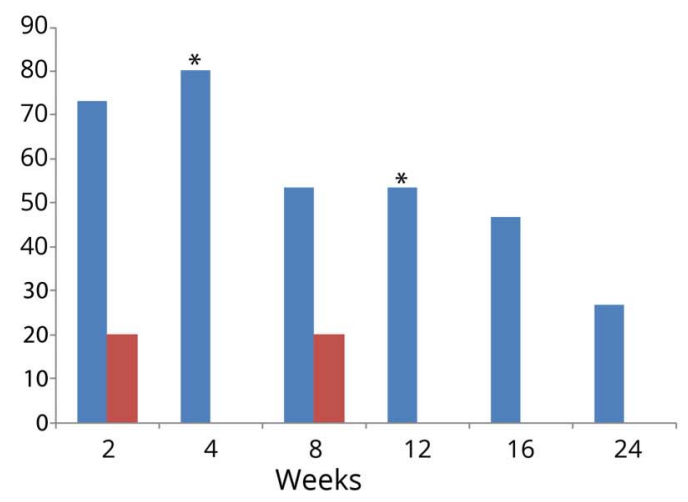

(A, B) ALSFRS-R least squares (LS) means of the change in slope (post-treatment minus pretreatment) for each of the post-treatment time points for the total population ( $A$ ) and rapid progressors (defined as those participants with a pretreatment ALSFRS-R change $\geq-2$ between screening and baseline) (B). The difference between the treated and placebo groups was statistically significant at the 2 and 4 weeks timepoints ( $p=0.021$ and 0.033 , respectively, indicated by $a$ * for $p<0.05)$. (C, D) The percentage of participants with a $\geq 1.5$-point improvement in the ALSFRS-R slope at the indicated time points as compared to their pretreatment slope over the $\sim 12$ weeks pretreatment period in the mesenchymal stem cell (MSC)-neurotrophic factor (NTF) cells treated and the placebo group total population (C) and rapid progressors (defined as participants with a pretreatment ALSFRS-R change $\geq-2$ between screening and baseline) (D). In the overall population, the difference was statistically significant at week $4(p=0.033)$. In rapid progressors, the differences between the treated and placebo groups were statistically significant at the 4 and 12 weeks timepoints $(p=0.004$ and 0.046 , respectively, indicated by a * for $p<0.05$ ).

(correlation $-0.77, p<0.0001)$, HGF and SDF-1a (correlation $-0.41, p=0.004)$, LIF and SDF-1 (correlation $-0.53, p=$ 0.005 ), and LIF and MCP-1 (correlation $-0.42, p=0.034)$ in MSC-NTF cells treated participants but not in the placebo group (data available on request).

A statistically significant correlation was observed between the decrease in MCP-1 levels post-transplantation and improvements in the post-transplantation compared to the pretransplantation slope. Significant correlations $(p<0.05)$ were identified at $4,12,16$, and 24 weeks post-treatment (figure 4B illustrates the correlation at 12 weeks; $p<0.01$ ), suggesting that higher CSF MCP-1 levels may be related to ALS disease progression, as measured by the ALSFRS-R.

\section{MiRNA}

We measured the expression of miR-34a, miR-132, miR-19, miR376-a, and miR-146a-5p, which are highly expressed in
MSC-NTF cells. ${ }^{4}$ We found these miRNAs to be increased post-treatment in the CSF of MSC-NTF-treated but not placebo participants. Interestingly, miR-34a, miR376-a, and miR-132 basal levels are notably lower in nonresponder as compared to responder participants. In addition, we measured the expression of miRNAs known to play an important role in ALS, including miR-9, miR-155, and miR-577. ${ }^{7-9}$ miR-155 and miR-577 were undetectable in the CSF of participants with ALS. miR-9, which is not expressed in MSC-NTF cells, did not significantly change in either group following treatment (data available on request).

\section{Data availability}

Appropriate anonymized data can be made available to qualified investigators upon submission of an acceptable analysis plan. Proposals should be directed to rkern@brainstorm-cell.com. To gain access, data requestors will need to sign a data access agreement. 

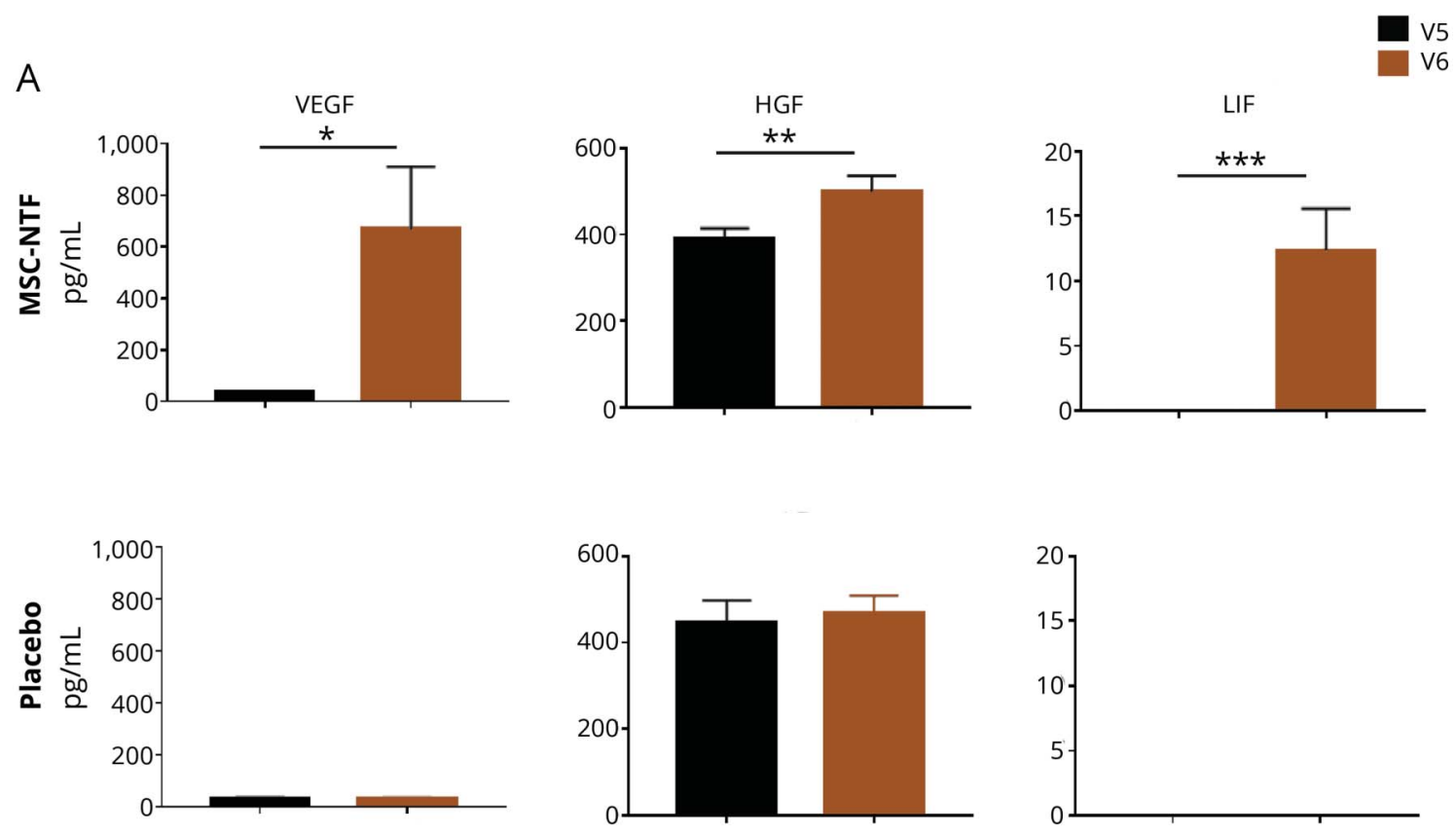

B
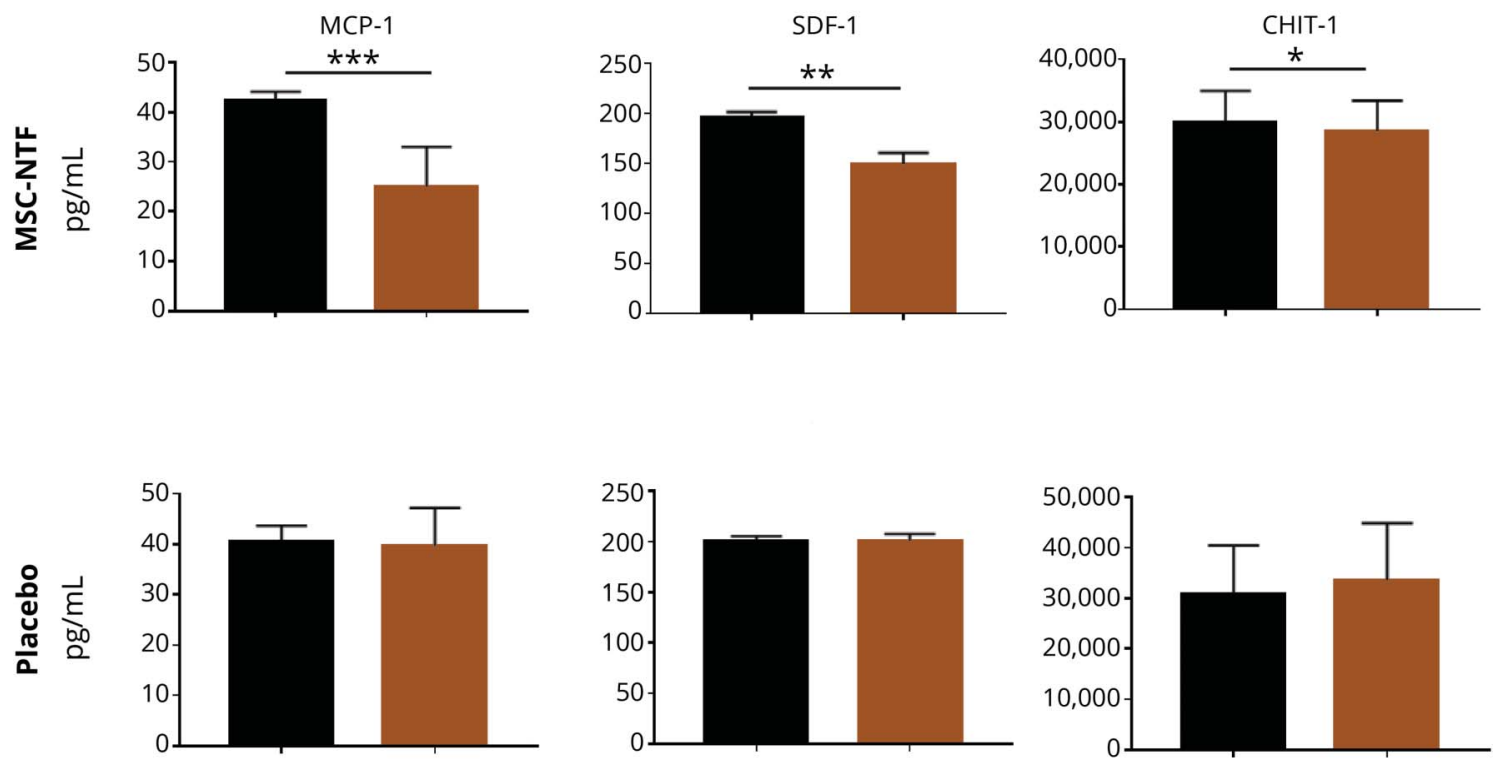

(A) A significant increase in vascular endothelial growth factor (VEGF), hepatocyte growth factor (HGF), and leukemia inhibitory factor (LIF) levels is shown in the CSF of the mesenchymal stem cell (MSC)-neurotrophic factor (NTF) cells treated group (upper panels) with no detectable change in the placebo group (lower panels). (B) A significant decrease in monocyte chemoattractant protein-1 (MCP-1), stromal cell-derived factor-1a (SDF-1), and chitotriosidase-1 (CHIT-1) levels is shown in the CSF of the MSC-NTF cells treated group (upper panels) with no significant change in the placebo group (lower panels). ${ }^{*} p<0.05,{ }^{*} p<<$ $0.01, * * * p<0.001$.

\section{Discussion}

This phase 2 clinical trial met its primary endpoint, demonstrating the safety of MSC-NTF cells delivered by combined IM and IT administration, and demonstrated promising effcacy, particularly in a prespecified subgroup of rapid progressors.
ALS is a heterogeneous disease; individual patients exhibit highly variable rates of functional decline. ${ }^{10}$ As a consequence, responder analyses may more accurately capture individual treatment responses ${ }^{11}$ and therefore provide a more informative comparison between treatment and placebo groups than changes in mean slope alone. We prespecified responder analyses using thresholds of $20 \%-30 \%$ improvement and 
Figure 4 Monocyte chemoattractant protein-1 (MCP-1) correlation to vascular endothelial growth factor (VEGF) secretion and to disease progression
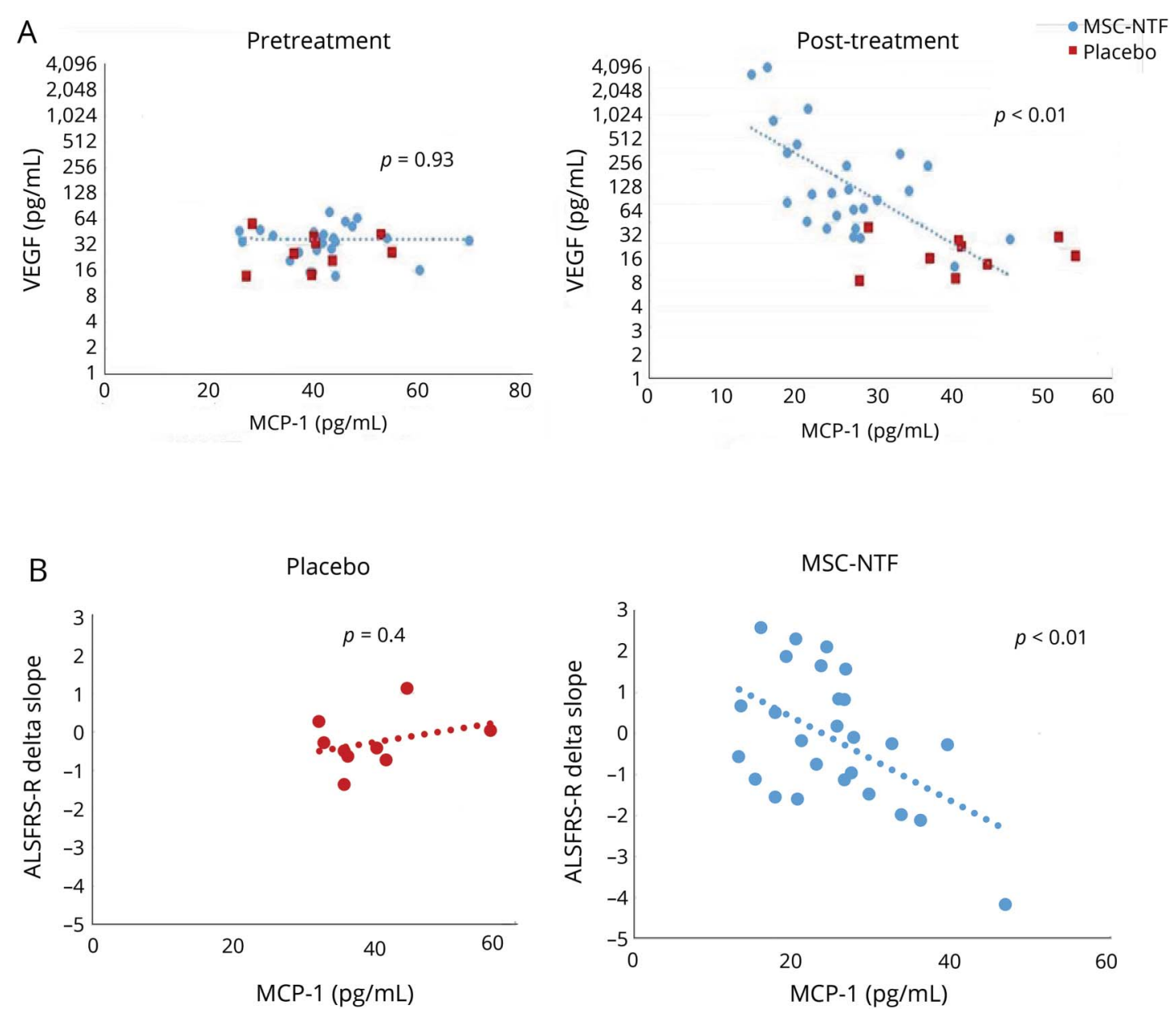

(A) A significant correlation between VEGF increase and MCP-1 decrease is shown in the CSF of the mesenchymal stem cell (MSC)-neurotrophic factor (NTF) cells treated group at visit six 2 weeks post-transplantation with no significant change in the placebo group. No correlation was seen between VEGF and MCP-1 levels prior to treatment (V5). (B) A significant correlation between MCP-1 in the CSF at 2 weeks post MSC-NTF cells treatment (visit 6, right panel) and a slower disease progression at 12 weeks post-treatment is shown, with no significant change in the placebo group (left panel). ALSFRS-R = Revised ALS Functional Rating Scale.

defined a range of thresholds for responders of $\geq 25 \%$ to $\geq 100 \%$ improvement at each follow-up visit. This is consistent with results of a survey of ALS clinicians and clinical researchers in which all participants endorsed a $25 \%$ or higher change in the ALSFRS-R slope as at least somewhat clinically meaningful, and $93 \%$ of the participants viewed a $50 \%$ change in decline as very clinically meaningful. ${ }^{12}$ In addition, the use of point change as a responder criterion precludes the exclusion of participants who are stable pretreatment (zero slope). The use of point and percentage change responder analyses thus provides a more robust and complete ALS efficacy estimate.

In the responder analyses, a higher proportion of MSC-NTFtreated participants achieved a $\geq 1.5$-point improvement per month, which may reflect disease stabilization. This improvement was most prominent immediately following transplantation and gradually decreased toward the end of the study, suggesting the need for repeated treatments to maintain a sustained therapeutic effect.

To mitigate the effect of disease heterogeneity and variability of ALSFRS-R slope change on efficacy outcomes, we performed a prespecified subgroup analysis based on the pretreatment ALSFRS-R overall score change $(<-2$ points or $\geq-2$ points). In the rapid progressors subgroup analysis $(<-2-$ point change), a statistically significant higher proportion of MSC-NTF-treated participants experienced a $\geq 1.5$ point/ month improvement compared to placebo.

We did not observe a significant difference in quantitative strength in the IM treated and untreated biceps and triceps muscles. This might reflect the ability to detect significant HHD changes in the small trial population, or asymmetrical rate of progression of each arm. The small sample size may have also reduced the likelihood of detecting changes in SVC 
given its known high test-retest variability in patients with ALS, in particular for those with bulbar onset. ${ }^{13}$ In addition, VC decline appears to be nonlinear in some patients with ALS who are slow SVC progressors and experience SVC decline only at a later stage of the disease, ${ }^{14}$ which may help explain the lack of treatment effect in this outcome measure.

Alternatively, a single dose may not have been sufficient to affect this outcome measure. Current clinical evaluation of MSC-NTF cells in ALS is focused on repeat dosing by the IT route of administration.

Preclinical and clinical studies suggest that MSCs tend to be short-lived and their beneficial effects may result from their capacity to regulate tissue homeostasis and inflammation via elaboration of an array of trophic factors. ${ }^{15}$ In this study, we observed a significant increase in the levels of MSC-NTF cells' specific factors (e.g., VEGF, HGF, and LIF) and several miRNAs in the CSF of treated participants. This supports the proposed paracrine mechanism of action of MSC-NTF cells in ALS, although we cannot rule out the possibility that the observed changes may represent a secondary mechanism induced by the cells.

Neuroinflammation is a prominent pathologic change at regions of motor neuron injury in ALS and is accompanied by microglial activation, astrogliosis, and the release of proinflammatory cytokines. ${ }^{16}$ CXCR4 and its ligand SDF-1 (CXCL12) are key regulators of microglia migration and recruitment. CXCL12/CXCR4 signaling in glial networks plays a major role in enhancing and maintaining spinal neuroinflammation. ${ }^{17,18}$ MCP-1, a potent chemoattractant and activating peptide, is expressed in astrocytes, microglia, and macrophages, is elevated in the CSF and spinal cord of patients with ALS, and may be associated with more severe disease and rapid progression. ${ }^{19}$

The significant decrease in MCP-1 and SDF-1 in MSC-NTFtreated participants, the strong correlation between cellspecific NTF increase and inflammatory biomarker decrease, and the relationship between CSF inflammatory biomarker reduction and the observed clinical improvement in the posttransplantation ALSFRS-R slope indicate that the cells may also act by modulating neuroinflammation.

miRNAs are small noncoding RNAs that regulate a wide variety of biological processes via RNA-dependent posttranscriptional silencing mechanisms. ${ }^{20}$ Extensive downregulation of miRNAs has been reported in spinal cord and motor neurons from patients with ALS. ${ }^{21}$ We have shown an increase in several cell-secreted miRNAs in treated but not placebo participants. The increase of miR-146a, known to mediate suppression of inflammatory response and to reduce MCP-1 levels, ${ }^{22}$ in responder participants and to a lesser extent in nonresponder participants, is consistent with the observed correlation between post-transplantation decrease in CSF MCP-1 and the observed reduction in disease progression in MSC-NTF-treated participants, further supporting the cells' anti-inflammatory mechanism of action. The increase in miR-132-3p, which is highly enriched in neurons, promotes neuronal outgrowth in vitro and in vivo, and regulates brain vascular integrity, provides another potential molecular mechanism relevant to the biological effects of MSC-NTF cells in ALS. ${ }^{23}$

Taken together, these exploratory analyses of NTF, inflammatory biomarker, and microRNA expression levels demonstrate for the first time the biological effects of MSCNTFs in patients with ALS in a randomized clinical trial. These results support the proposed paracrine mechanism of action of MSC-NTFs and the combined effects on both neuroprotection and neuroinflammation. We observed increased expression of the neuroprotection markers VEGF, HGF, LIF, miR-132p, and miR-376 and a decreased expression of neuroinflammatory markers MCP-1 and SDF-1, as well as an increase in miR-146, further supporting the hypothesis that MSC-NTFs operate in study participants with ALS to both increase neuroprotection and decrease neuroinflammation. It is highly likely that these 2 interrelated mechanisms may be synergistic.

This randomized, double-blind, placebo-controlled phase 2 trial of MSC-NTF cell transplant showed that administration of bone-marrow-derived, autologous MSC-NTF cells is safe and well-tolerated. In addition, we have demonstrated significant efficacy in a prespecified subpopulation of rapid progressors, where meaningful changes in post-transplant ALSFRS-R slope change were observed. The stabilization or reversal of ALSFRS-R score in rapid progressors establishes a clear step forward for the phase III repeated-dose trial in this predefined ALS subgroup.

\section{Author contributions}

James D. Berry: drafting/revising the manuscript, data acquisition, study concept or design, analysis or interpretation of data, accepts responsibility for conduct of research and final approval. Merit E. Cudkowicz: drafting/revising the manuscript, analysis or interpretation of data, accepts responsibility for conduct of research and final approval, acquisition of data, study supervision. Anthony J. Windebank: drafting/revising the manuscript, data acquisition, study concept or design, analysis or interpretation of data, accepts responsibility for conduct of research and final approval, study supervision. Nathan P. Staff: drafting/revising the manuscript, data acquisition, study concept or design, analysis or interpretation of data, accepts responsibility for conduct of research and final approval, study supervision. Margaret Owegi: data acquisition, accepts responsibility for conduct of research and final approval, acquisition of data. Katherine Nicholson: drafting/revising the manuscript, accepts responsibility for conduct of research and final approval, acquisition of data, study supervision. Diane McKenna-Yasek: data acquisition, accepts responsibility for conduct of research and final approval, acquisition of data, study supervision. Yossef S. Levy: analysis or interpretation of 
data, accepts responsibility for conduct of research and final approval, contribution of vital reagents/tools/patients. Natalie Abramov: analysis or interpretation of data, accepts responsibility for conduct of research and final approval, acquisition of data. Haggai Kaspi: drafting/revising the manuscript, analysis or interpretation of data, accepts responsibility for conduct of research and final approval, acquisition of data. Munish Mehra: drafting/revising the manuscript, analysis or interpretation of data, accepts responsibility for conduct of research and final approval, statistical analysis. Revital Aricha: drafting/revising the manuscript, analysis or interpretation of data, accepts responsibility for conduct of research and final approval, acquisition of data. Yael Gothelf: drafting/revising the manuscript, study concept or design, analysis or interpretation of data, accepts responsibility for conduct of research and final approval, study supervision. Robert H. Brown: drafting/ revising the manuscript, data acquisition, study concept or design, analysis or interpretation of data, accepts responsibility for conduct of research and final approval, acquisition of data, study supervision.

\section{Study funding}

Study supported by Brainstorm Cell Therapeutics.

\section{Disclosure}

J. Berry has no financial conflicts; has acted as a consultant for an advisory panel for Denali Therapeutics; and has research funding and/or acts as an investigator for studies funded by MT Pharma, Brainstorm Cell Therapeutics, Amylyx Pharmaceuticals, Genentech, Anelixis Pharmaceuticals, ALS Association, Muscular Dystrophy Association, ALS Finding a Cure, and ALS One. M. Cudkowicz is a consultant for Biohaven, Takeda, MT pharma, Aclipse and Avexis; was on a DSMB for Lilly; and receives grant support from NINDS, ALSFAC, and the ALS Association. A. Windeank is a consultant for the Federal Trade Commission on stem cell issues (those consulting fees go to his research budget at Mayo Clinic); and serves on the external advisory boards of 2 research institutes in Europe: CURAM in Ireland and SCITReCS in Austria (he derives no compensation for these positions other than for travel and accommodation). N. Staff has no financial conflicts; has research funding and/or acts as an investigator for studies funded by Orion Pharmaceuticals, Cytokinetics, Regenerative Medicine Minnesota, and Brainstorm Therapeutics; and has research funding from the NIH (R01 CA 211887). M. Owegi has no financial conflicts with Brainstorm and no relevant financial interests/disclosures. K. Nicholson has no financial conflicts with the Brainstorm Study; has acted as a consultant for an advisory panel for Avanir Pharmaceuticals; and has research funding and/or acts as an investigator for studies funded by Brainstorm Cell Therapeutics, Amylyx Pharmaceuticals, ALS Association, Muscular Dystrophy Association, ALS Finding a Cure, Target ALS, and the Salah Foundation. D. McKenna-Yasek has no conflict of interests regarding the Brainstorm Therapeutics clinical trial. Y.S. Levy is a Brainstorm employee. N. Abramov is a Brainstorm employee. H. Kaspi is a Brainstorm employee. M. Mehra received consulting fees for the analyses from Brainstorm Cell Therapeutics. R. Aricha is a Brainstorm employee. Y. Gothelf is a Brainstorm employee. R. Brown has no financial conflicts with the Brainstorm study; is a scientific founder of Apic-Bio and holds equity in Amlyx and Imstar; and has research funding and/or acts as an investigator without personal financial compensation for studies funded by Brainstorm Cell Therapeutics, the Angel Fund, ALS Association, Muscular Dystrophy Association, ALS Finding a Cure, Project ALS, Target ALS, and ALS One. Go to Neurology.org/ $\mathrm{N}$ for full disclosures.

\section{Publication history}

Received by Neurology January 30, 2019. Accepted in final form July 30, 2019.

\section{References}

1. Brown RH, Al-Chalabi A. Amyotrophic lateral sclerosis. N Engl J Med 2017;377: 162-172.

2. Uccelli A, Moretta L, Pistoia V. Mesenchymal stem cells in health and disease. Nat Rev Immunol 2008;8:726-736.

3. Gothelf Y, Abramov N, Harel A, Offen D. Safety of repeated transplantations of neurotrophic factors-secreting human mesenchymal stromal stem cells. Clin Transl Med 2014;3:21.

4. Gothelf $\mathrm{Y}$, Kaspi H, Abramov N, Aricha R. miRNA profiling of NurOwn': mesenchymal stem cells secreting neurotrophic factors. Stem Cell Res Ther 2017;8:249.

5. Petrou P, Gothelf Y, Argov Z, et al. Safety and clinical effects of mesenchymal stem cells secreting neurotrophic factor transplantation in patients with amyotrophic lateral sclerosis: results of phase 1/2 and 2a clinical trials. JAMA Neurol 2016;73:337-344.

6. Brooks BR, Miller RG, Swash M, Munsat TL. El Escorial revisited: revised criteria for the diagnosis of amyotrophic lateral sclerosis. Amyotroph Lateral Scler Other Motor Neuron Disord 2000;1:293-299.

7. Zhang Z, Almeida S, Lu Y, et al. Downregulation of microRNA-9 in iPSC-derived neurons of FTD/ALS patients with TDP-43 mutations. PLoS One 2013;8:e76055.

8. Butovsky O, Jedrychowski MP, Cialic R, et al. Targeting miR-155 restores abnormal microglia and attenuates disease in SOD1 mice. Ann Neurol 2015;77:75-99.

9. Figueroa-Romero C, Hur J, Lunn JS, et al. Expression of microRNAs in human postmortem amyotrophic lateral sclerosis spinal cords provides insight into disease mechanisms. Mol Cell Neurosci 2016;71:34-45.

10. Kiernan MC, Vucic S, Cheah BC, et al. Amyotrophic lateral sclerosis. Lancet 2011; 377:942-955

11. Finkel RS, Mercuri E, Darras BT, et al. Nusinersen versus sham control in infantileonset spinal muscular atrophy. N Engl J Med 2017;377:1723-1732.

12. Castrillo-Viguera C, Grasso DL, Simpson E, Shefner J, Cudkowicz ME. Clinical significance in the change of decline in ALSFRS-R. Amyotroph Lateral Scler 2010;11: $178-180$

13. Banno H, Schoenfeld D, Cudkowicz ME, Atassi N. Vital capacity: high test-retest variability in ALS patients with bulbar weakness. J Neurol Sci 2017:381.

14. Polkey MI, Lyall RA, Yang K, Johnson E, Leigh PN, Moxham J. Respiratory muscle strength as a predictive biomarker for survival in amyotrophic lateral sclerosis. Am J Respir Crit Care Med 2017;195:86-95.

15. Wang Y, Chen X, Cao W, Shi Y. Plasticity of mesenchymal stem cells in immunomodulation: pathological and therapeutic implications. Nat Immunol 2014;15 1009-1016.

16. Zhao W, Beers DR, Appel SH. Immune-mediated mechanisms in the pathoprogression of amyotrophic lateral sclerosis. J Neuroimmune Pharmacol 2013;8:888-899.

17. Rabinovich-Nikitin I, Ezra A, Barbiro B, Rabinovich-Toidman P, Solomon B. Chronic administration of AMD3100 increases survival and alleviates pathology in SOD1(G93A) mice model of ALS. J Neuroinflammation 2016;13:123.

18. Bezzi P, Domercq M, Brambilla L, et al. CXCR4-activated astrocyte glutamate release via TNFalpha: amplification by microglia triggers neurotoxicity. Nat Neurosci 2001;4: $702-710$.

19. Guo J, Yang X, Gao L, Zang D. Evaluating the levels of CSF and serum factors in ALS Brain Behav 2017;7:e00637.

20. Bartel DP. MicroRNAs: genomics, biogenesis, mechanism, and function. Cell 2004 116:281-297.

21. Emde A, Eitan C, Liou LL, et al. Dysregulated miRNA biogenesis downstream of cellular stress and ALS-causing mutations: a new mechanism for ALS. EMBO J 2015; 34:2633-2651.

22. Roos J, Enlund E, Funcke JB, et al. miR-146a-mediated suppression of the in flammatory response in human adipocytes. Sci Rep 2016;6:38339.

23. Xu B, Zhang Y, Du XF, et al. Neurons secrete miR-132-containing exosomes to regulate brain vascular integrity. Cell Res 2017;27:882-897. 


\section{Neurology}

\section{NurOwn, phase 2, randomized, clinical trial in patients with ALS: Safety, clinical, and biomarker results}

James D. Berry, Merit E. Cudkowicz, Anthony J. Windebank, et al.

Neurology 2019;93;e2294-e2305 Published Online before print November 18, 2019

DOI 10.1212/WNL.0000000000008620

This information is current as of November 18, 2019

\section{Updated Information \& Services}

References

Citations

Subspecialty Collections

Permissions \& Licensing

Reprints including high resolution figures, can be found at: http://n.neurology.org/content/93/24/e2294.full

This article cites 22 articles, 1 of which you can access for free at: http://n.neurology.org/content/93/24/e2294.full\#ref-list-1

This article has been cited by 3 HighWire-hosted articles: http://n.neurology.org/content/93/24/e2294.full\#\#otherarticles

This article, along with others on similar topics, appears in the following collection(s):

Amyotrophic lateral sclerosis

http://n.neurology.org/cgi/collection/amyotrophic_lateral_sclerosis_ Anterior nerve cell disease http://n.neurology.org/cgi/collection/anterior_nerve_cell_disease Class I

http://n.neurology.org/cgi/collection/class_1 Clinical trials Randomized controlled ( $\overline{\mathbf{C}}$ ONSORT agreement) http://n.neurology.org/cgi/collection/clinical_trials_randomized_contro lled_consort_agreement

Information about reproducing this article in parts (figures,tables) or in its entirety can be found online at:

http://www.neurology.org/about/about_the_journal\#permissions

Information about ordering reprints can be found online: http://n.neurology.org/subscribers/advertise

Neurology ${ }^{\circledR}$ is the official journal of the American Academy of Neurology. Published continuously since 1951, it is now a weekly with 48 issues per year. Copyright Copyright (C) 2019 The Author(s). Published by Wolters Kluwer Health, Inc. on behalf of the American Academy of Neurology.. All rights reserved. Print ISSN: 0028-3878. Online ISSN: 1526-632X.

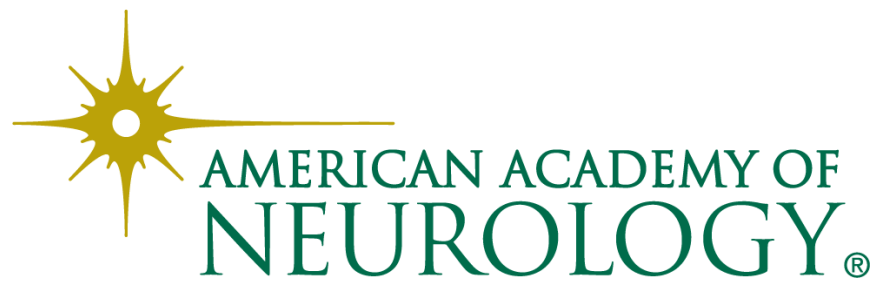

